\title{
SUPRIMENTO ARTERIAL PARA AS GLÂNDULAS ADRENAIS NO RATÃO-DO-BANHADO (Myocastor coypus Molina, 1782)
} (Arterial supply forto the adrenal glands of the nutria (Myocastor coypus Molina, 1782)

\author{
MACHADO, G.V. ${ }^{1}$; ROMAGNOLLI, P. ${ }^{2}$; SOUZA, J.R. ${ }^{1}$; \\ TURQUETI, V.S. ${ }^{2}$; ULIANA, S.M. ${ }^{2}$; SILVA, M.H. ${ }^{1}$
}

\author{
1Universidade Federal do Paraná / Campus Palotina Cx. Postal 54 \\ CEP. 85950-000 - Palotina/PR - E-mail: machadogv@yahoo.com.br \\ ${ }^{2}$ Universidade Paranaense - UNIPAR
}

RESUMO - Foram estudados os componentes vasculares arteriais destinados à irrigação sangüínea das glândulas adrenais de 10 exemplares adultos, machos e fêmeas, de ratãodo-banhado (Myocastor coypus). Esses animais foram injetados, após morte natural, através da artéria carótida comum, com solução corada de Neoprene látex, em seguida foram fixados em solução aquosa de formol a $10 \%$, mantidos em recipientes contendo a mesma solução, por período mínimo de 72 horas, e dissecados. As dissecações permitiram observar que: a) a glândula adrenal direita foi atingida por ramos colaterais provenientes das artérias frênica caudal direita $(50 \%)$, abdominal cranial direita $(30 \%)$, renal direita $(30 \%)$, primeira lombar (30\%) e aorta abdominal $(30 \%)$; b) para a glândula adrenal esquerda foram destinados colaterais provenientes das artérias renal esquerda $(80 \%)$, aorta abdominal $(50 \%)$, abdominal cranial esquerda $(20 \%)$ e segunda lombar $(10 \%)$.

Palavras chave: anatomia, glândulas adrenais, ratão-do-banhado, nutria.

ABSTRACT - An anatomical study has been carried out on the arterial branching system for nutria (Myocastor coypus Molina, 1782) adrenal glands blood supply. After natural death, a total of 10 male and female adult nutrias were injected through the carotid artery with a colored solution of Neoprene latex. Soon after this step they were maintained for a period of 72 hours of fixation in a 10\% aqueous solution of formalin, being dissected right after this procedure. The following observations were then carried out: a) the right adrenal gland is supplied by branches of the right caudal phrenic artery $(50 \%)$, right cranial abdominal artery $(30 \%)$, right renal artery $(30 \%)$, first lumbar artery $(30 \%)$, and abdominal aorta $(30 \%)$; b) the left adrenal gland is supplied by branches from the left renal artery $(80 \%)$, abdominal aorta $(50 \%)$, left cranial abdominal artery $(20 \%)$ and the second lumbar artery $(10 \%)$.

Key words: anatomy, adrenal glands, nutria.

\section{Introdução}

\section{A família Capromyidae (RODENTIA:} MAMMALIA), possui três gêneros já extintos, restando apenas, no Brasil, o gênero Myocastor, com uma única espécie, o $M$. coypus, conhecida como ratão-do-banhado, ou nutria. Trata-se de um mamífero roedor, muito perseguido pelo homem, que visa o aproveitamento de sua pele e carne (SILVA, 1994). Embora originário do extremo sul do continente americano, esse animal foi introduzido, em meados do último século, nos EUA e Europa, com vistas à sua exploração econômica tendo, entretanto, se dispersado na natureza.

Visando a obtenção de informações, de cunho morfológico, que possam fundamentar procedimentos que visem a preservação do nutria na natureza, bem como subsidiar pesquisas que busquem aprimorar métodos de sua exploração racional, o presente trabalho, como parte de uma linha de pesquisa, mira aspectos relacionados à irrigação sangüínea das glândulas adrenais desses animais.

Apesar da importância do nutria como 
componente da biodiversidade, bem como do seu potencial como animal passível de exploração econômica, haja vista o valor de sua pele e a qualidade de sua carne, aliados ao fato de sua alta prolificidade e capacidade de adaptação - sem perder de vista o interesse da anatomia comparativa - poucas são as referências encontradas na literatura a respeito de sua morfologia, referências essas restritas a informações gerais sobre a sua dentição, coloração da pele e volume corporal (SILVA, 1994).

No atinente ao suprimento vascular arterial para as glândulas adrenais desses animais, nenhuma referência foi encontrada. Entretanto, o tema é objeto de diferentes formas de abordagens, para outras espécies de roedores e lagomorfas, como em GREENE (1955) e FARRIS e GRIFFITH (1963) que, referindo-se ao rato, afirmam que as artérias renais, antes de atingirem o hilo renal, originam ramos supra-renais inferiores. Reportam-se ainda a uma artéria supra-renal superior, que surge como ramo da artéria frênica inferior. De maneira bastante semelhante, COOK (1965) relata, em camundongos de laboratório, uma artéria supra-renal superior esquerda, oriunda da aorta abdominal, assim como registra uma artéria supra-renal inferior esquerda, proveniente ora da artéria frênica inferior, ora da artéria renal esquerda.

Referindo-se ao coelho, BARONE et al. (1973) fazem alusão aos ramos suprarenais, como ramos provindos das artérias renais, aorta abdominal, abdominal cranial, frênica caudal e lombares I e II.

Ao investigarem a ramificação da aorta abdominal no hamster, ORSI et al. (1975) descreveram um vaso delgado surgindo junto ao terço médio da artéria renal esquerda, enviando um de seus ramos, que denominaram artéria adrenal cranial, para a glândula adrenal daquele mesmo lado. PIFFER et al. (1980) registraram as artérias adrenais cranial e caudal em ratos, como responsáveis pela irrigação sangüínea daquelas glândulas.

HEBEL e STROMBERG (1986) afiançam que, em ratos de laboratório, as glândulas adrenais são irrigadas pelas artérias supra-renal cranial, proveniente da aorta, e supra-renal caudal, oriunda da artéria renal. Por outro lado, SANTOS et al. (1991), estudando a irrigação sangüínea das glândulas adrenais de coelhos, afirmam que a glândula esquerda é atingida por ramos das artérias renal esquerda, aorta abdominal e mesentérica cranial. Porém a glândula adrenal direita, além das artérias já citadas, recebe ramos ainda da primeira artéria lombar.

MIZUKAMI e TANAKA (1992), referindo-se ao rato, descrevem ainda uma artéria supra-renal, originando-se da artéria frênica inferior, como responsável pela irrigação da glândula adrenal. Entretanto, MACHADO et al. (1999), em trabalho realizado em treze lebres (Lepus europaeus Pallas, 1778), anunciam que a glândula adrenal direita é atingida por ramos das artérias abdominal cranial direita, renal direita, frênica caudal direita, aorta abdominal e celíaca, nesta ordem de participação, ao passo que a glândula esquerda é atingida por ramos provenientes das artérias abdominal cranial esquerda, renal esquerda, aorta abdominal e mesentérica caudal, nesta ordem de participação.

\section{Material e Método}

Para a realização do presente trabalho, utilizaram-se 10 exemplares adultos de ratão-do-banhado (Myocastor coypus), sendo seis machos e quatro fêmeas, com diferentes hiatóricos de óbito. Esses animais, em laboratório, tiveram a sua artéria carótida comum canulada, por onde injetou-se solução corada de Neoprene látex ${ }^{*}$, até que se confirmasse a total repleção de todo o seu sistema arterial. A seguir foram fixados em solução aquosa de formol a $10 \%$, mantidos em recipientes contendo solução semelhante, onde permaneceram por período mínimo de 72 horas, e dissecados.

As dissecações foram realizadas a partir de uma incisão mediana na parede do abdome, seguida da incisão do hipocôndrio esquerdo, resultando assim o

\footnotetext{
* Dupont do Brasil S/A.
}

Archives of Veterinary Science v.7, n.2, p.9-14, 2002 
acesso à cavidade peritoneal. Tal procedimento permitiu visualizar a aorta abdominal e seus principais ramos, particularmente aqueles destinados às glândulas adrenais. Após a identificação daqueles ramos arteriais, confeccionaramse esquemas representativos das diferentes disposições vasculares, os quais constituíram objeto de análise e documentação.

\section{Resultados}

Ao analisarem-se as disposições vasculares arteriais, particularmente relacionadas às glândulas adrenais do ratão-do-banhado (Myocastor coypus), verificou-se uma forte assimetria entre essas glândulas, em especial quanto à sua forma, onde a adrenal direita apresenta-se em formato triangular e de maior volume, quando comparada com a glândula esquerda, que se apresenta com menor volume e com formato alongado. Essas glândulas, que são de grande volume relativo, localizam-se junto à extremidade cranial dos rins.

A glândula adrenal direita ocupa posição mais cranial em relação à glândula esquerda, sendo que os ramos vasculares que a atingem são provenientes das artérias frênica caudal direita, renal direita, abdominal cranial direita, primeira lombar e aorta abdominal. Ao passo que a glândula adrenal esquerda recebe, como suprimento arterial, ramos provenientes das artérias renal esquerda, aorta abdominal, abdominal cranial esquerda e segunda lombar.

A artéria frênica caudal direita enviou, em cinco casos $(50 \%)$, ramos colaterais para a glândula adrenal direita, contribuindo com um ramo em quatro animais $(40 \%)$ e com dois ramos em outro caso (10\%). Nestes cinco casos, a glândula adrenal direita foi abordada simultaneamente por ramos da artéria abdominal cranial, em dois casos (20\%), da primeira artéria lombar, em um caso $(10 \%)$, da artéria renal direita em dois casos $(20 \%)$, e da aorta abdominal em dois outros casos (20\%).
A artéria abdominal cranial direita enviou, em três casos (30\%), um ramo colateral para a irrigação da glândula adrenal direita, sendo que em dois desses casos $(20 \%)$ em conjunto com ramos das artérias frênica caudal e aorta abdominal; no outro caso (10\%), porém, participou em conjunto com a artéria renal direita.

Por seu turno, a artéria renal direita participou, em três casos (30\%), na irrigação sangüínea da glândula adrenal direita, sendo com três ramos em um animal $(10 \%)$ e dois ramos nos dois outros casos (20\%). Em um caso (10\%), associou-se à artéria renal um ramo proveniente da artéria abdominal cranial. Em um dos animais estudados (10\%), onde a glândula adrenal direita recebeu dois ramos oriundos da artéria renal direita, houve a participação ainda de um ramo proveniente da primeira artéria lombar. Esta última artéria esteve presente na irrigação da glândula adrenal direita em três dos animais estudados $(30 \%)$, sendo que em dois casos $(20 \%)$, associou-se à aorta, com um ramo cada; no outro caso (10\%) sua participação foi em conjunto com um ramo da artéria frênica caudal.

Através de ramos diretos, a aorta abdominal contribuiu para a irrigação da glândula adrenal direita em três animais $(30 \%)$, sendo que em dois desses casos (20\%) em conjunto com um ramo da primeira artéria lombar; em outro caso $(10 \%)$, o ramo direto da aorta foi acompanhado por ramos das artérias frênica caudal e abdominal cranial, sendo um ramo de cada.

Para a irrigação sangüínea da glândula adrenal esquerda, a artéria renal esquerda participou em oito casos (80\%), sendo por intermédio de um ramo em cinco casos $(50 \%)$ e de dois ramos em três casos (30\%). Essa artéria atuou em conjunto com a aorta em três casos (30\%), com a artéria abdominal cranial em dois exemplares estudados $(20 \%)$ e com a segunda artéria lombar em um outro caso (10\%). Em três ocasiões $(30 \%)$, a artéria renal esquerda apresentou-se como única responsável por emitir ramos para a glândula adrenal esquerda. 
A aorta abdominal, em cinco oportunidades $(50 \%)$, enviou um ramo direto para a glândula adrenal esquerda, sendo que, em dois desses casos $(20 \%)$, de maneira exclusiva; nos três casos restantes $(30 \%)$, porém, em conjunto com ramos da artéria renal esquerda (dois casos - 20\%) e da artéria abdominal cranial esquerda (um caso $-10 \%)$.

FIGURA 1 - DESENHO ESQUEMÁTICO REPRESENTATIVO DE ALGUMAS FORMAS DE ABORDAGEM VASCULAR ARTERIAL PARA AS GLÂNDULAS ADRENAIS NO RATÃO-DO-BANHADO (myocastor coypus), EM VISTA VENTRAL. 1 - AORTA ABDOMINAL; 2 - A. CELÍACA; 3 - A. MESENTÉRICA CRANIAL; 4 - A. RENAL DIREITA; 5 - A. RENAL ESQUERDA; 6 - TRONCO FRÊNICO-ABDOMINAL; 7 - A. ABDOMINAL CRANIAL ESQUERDA; 8 - A. FRÊNICA DIREITA; 9, 10, 11 E 12 RAMOS ADRENAIS; A - GLÂNDULAS ADRENAIS; RD - RIM DIREITO; RE - RIM ESQUERDO. PALOTINA, 2002.

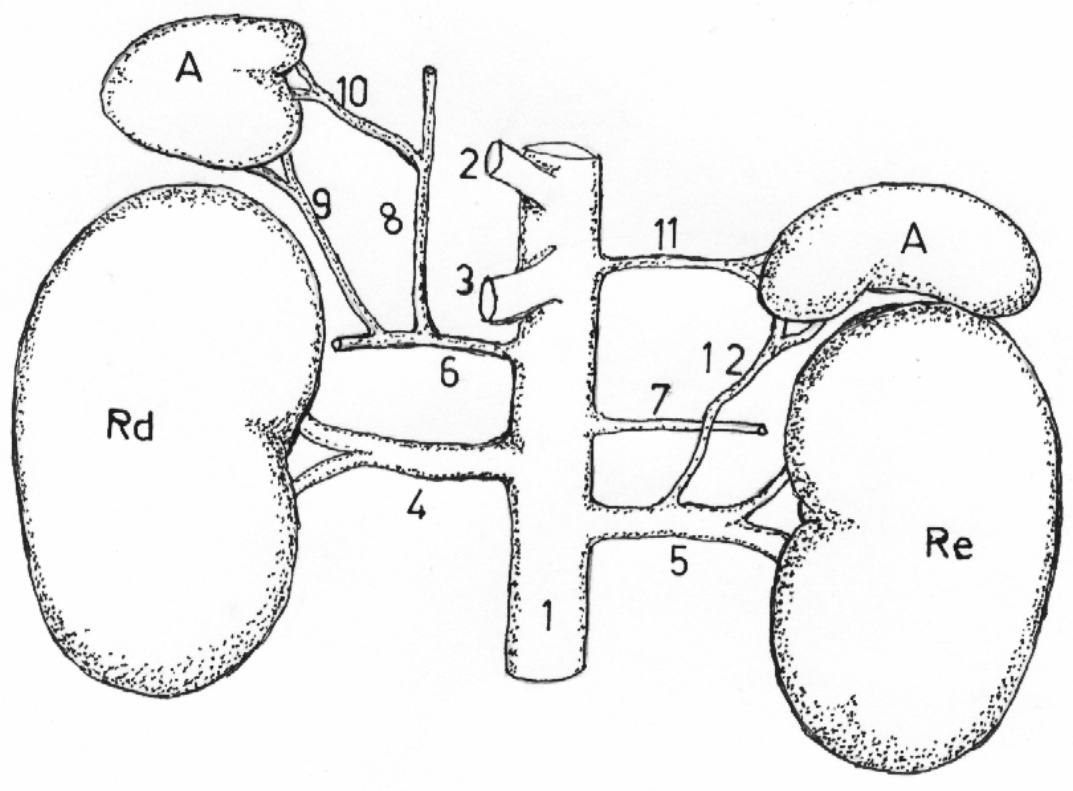

A artéria abdominal cranial esquerda contribuiu para a irrigação da glândula adrenal esquerda em dois animais $(20 \%)$, sendo que em um dos casos $(10 \%)$ em conjunto com ramos das artérias renal esquerda e aorta abdominal; no outro espécime examinado, a artéria abdominal cranial esquerda, que emitiu um ramo para aquela glândula, foi auxiliada por dois ramos da artéria renal esquerda. Em uma oportunidade $(10 \%)$, um ramo proveniente da segunda artéria lombar, em conjunto com um ramo da artéria renal esquerda, destinou-se à glândula adrenal esquerda.

\section{Discussão}

Os achados relacionados às diferentes formas de abordagem vascular arterial, ora relatados no ratão-do-banhado, permitem algumas considerações, quando comparados àqueles referentes a outros roedores e lagomorfas, encontrados na literatura. Em GREENE (1955) e FARRIS e GRIFFITH (1963), reportando-se ao rato, as artérias destinadas à irrigação das glândulas adrenais, que esses autores denominam ramos supra-renais inferiores, têm sua origem nas artérias renais. Esses mesmos autores referem-se ainda às artérias supra-renais superiores, como 
Suprimento arterial para as glândulas adrenais no Ratão-do-banhado (Myocastor coypus Molina, 1782)

ramos da artéria frênica inferior, o que é referendado por MIZUKAMI e TANAKA (1992), para o rato, e por COOK (1965), desta vez em camundongos. Entretanto, este último autor acrescenta uma artéria supra-renal superior esquerda, proveniente da aorta. Os dados ora encontrados no nutria confirmam a participação daqueles vasos, embora não adotando a nomenclatura utilizada pelos autores, porém registram ainda outras origens para os ramos adrenais.

HEBEL e STROMBERG (1986), referindo-se ao rato, enfatizam a origem das artérias supra-renais cranial e caudal, como ramos das artérias aorta e renal, respectivamente. PIFFER et al. (1980) endossam essas afirmativas, porém não se detêm à origem daqueles vasos. Esses vasos estão confirmados no nutria, porém não de forma excludente pois, como visto, outras artérias também se incumbem da emissão de ramos adrenais. Entretanto, quanto a denomina-los craniais e caudais, optou-se por não faze-lo, haja vista a existência de ramos, às vezes no mesmo animal, que então deveriam ser reconhecidos como médios e, ainda assim, com diferentes disposições.

Os dados relativos ao coelho, acrescentados por SANTOS et al. (1991), dão conta de que a glândula adrenal esquerda daqueles animais é atingida por ramos das artérias renal esquerda, aorta abdominal e mesentérica cranial. Já para a glândula direita afluem ainda ramos da primeira artéria lombar. Esses dados se coadunam em parte com os registros ora anunciados, pois no nutria não houve a participação da artéria mesentérica cranial, mas acrescentaram-se as contribuições das artérias abdominais craniais, frênicas caudais e segunda lombar.

Quanto aos dados de MACHADO et al. (1999), relativos à lebre, quando comparados aos que ora se registram no ratão-do-banhado, notam-se convergências entre os mesmos, exceto pela ausência de participação, neste último, das artérias celíaca e mesentérica cranial, assim como pela presença, também no ratão-dobanhado, de ramos adrenais provenientes da primeira e segunda artérias lombares.

\section{Conclusões}

Com base nos achados ora anunciados, pode-se afirmar que, no ratão-do-banhado (Myocastor coypus), a forma de abordagem vascular arterial das glândulas adrenais difere das descrições já realizadas em outros roedores e lagomorfas. Ressalte-se que para a glândula adrenal direita destinam-se ramos provenientes das artérias frênica caudal direita, abdominal cranial direita, renal direita, primeira artéria lombar e aorta abdominal. Já para a glândula adrenal esquerda orientam-se ramos oriundos das artérias renal esquerda, aorta abdominal, abdominal cranial esquerda e segunda artéria lombar.

\section{Referências}

BARONE, R.; PAVAUX, C.; BLIN, P.C.; CUQ, $P$. Atlas d'anatomie du lapin. Paris: Masson, 1973. p. 129-186.

COOK, M.J. The anatomy of the laboratory mouse. New York: Academic Press, 1965. p. 122-131.

FARRIS, E.J.; GRIFFITH, J.A. The rat in laboratory investigation. 2. ed. New York: Hafner Publishing Company, 1963. p. 46.

GREENE, E.C. Anatomy of the rat. New York: Hafner Publishing Company, 1955, p. 73.

HEBEL, R.; STROMBERG, M.W. Anatomy and embriology of the laboratory rat. London: BioMed Verlag Wörthsee, 1986. p. 106-109.

MACHADO, G.V.; SILVA, F.O.C.; MIGLINO, M.A.; SANTOS, T.C.; ANDRIOLI, L.G. Suprimento arterial para as glândulas adrenais em Lepus europaeus Pallas, 1778 - LAGOMORPHA: MAMMALIA. Arquivo de Ciências Veterinárias e Zoologia da UNIPAR, Umuarama, v. 2, n. 1, p. 23-27, 1999.

MIZUKAMI, S.; TANAKA, S. A macroscopical study of the inferior frenic artery of female rats, with reference to the embriological background of ocurrence of the genital artery from this artery. Okajima, Folia Anatomica Japonica, Tokyo, v. 69, n. 1, p. 1-10, 1992. 
ORSI, A.M.; SILVA, P.P.; FERNANDES, W.A. Estudo de los ramos de la aorta abdominal del hamster dourado (Mesocricetos auratus). Madrid, Archives of Anatomy and Embryology, v. 12, p. 5, 1975.

PIFFER, C.R.; GARCIA, P.J.; SOARES, J.C.; ZORZETTO, J.C. Origen of the cranial and caudal adrenal arteries in the rat (Rattus norvegicus). Anatomia Histologia Embryologia, Berlin, v. 9, n. 4, p. 325-358, 1980.

Recebido para publicar: $\quad$ 06/05/2002

Aprovado:

$28 / 10 / 2002$
SANTOS, A.L.Q.; SEVERINO, R.S.; SILVA, F.O.C.; DRUMMOND, S.S.; BOMBONATO, P.P.; PEREIRA, C.C.H.; APPOLINÁRIO, A.V.M. Irrigação das glândulas adrenais em coelhos sem raça definida. In: CONGRESSO LUSO-BRASILEIRO DE ANATOMIA, 8; CONGRESSO BRASILEIRO DE ANATOMIA, 14., 1991, São Paulo. Anais... São Paulo: Sociedade Brasileira de Anatomia, 1991. p. 93.

SILVA, F. Mamíferos silvestres do Rio Grande do Sul. 2. ed. Porto Alegre: Fundação Zoobotânica do Rio Grande do Sul, 1994, p. 122. 